\title{
STEADY-STATE PHARMACOKINETICS OF METFORMIN IN OBESE PATIENTS WITH TYPE 2 DIABETES MELLITUS: A PRELIMINARY STUDY
}

\author{
NINGRUM VDA ${ }^{1,2 *}$, IKAWATI Z ${ }^{2}$, SADEWA AH ${ }^{3}$, IKHSAN MR ${ }^{4}$, SAEPUDIN ${ }^{1}$ \\ ${ }^{1}$ Department of Pharmacy, Universitas Islam Indonesia, Yogyakarta, Indonesia. ${ }^{2}$ Department of Pharmacology and Clinical Pharmacy, \\ Faculty of Pharmacy, Universitas Gadjah Mada, Yogyakarta, Indonesia. ${ }^{3}$ Department of Biochemistry, Faculty of Medicine, Universitas \\ Gadjah Mada, Yogyakarta, Indonesia. ${ }^{4}$ Department of Internal Medicine, Dr. Sardjito Hospital, Yogyakarta, Indonesia. \\ Email: vitarani.ningrum@uii.ac.id
}

Received: 19 September 2016, Revised and Accepted: 29 September 2016

\section{ABSTRACT}

Objective: This study aimed to determine the metformin plasma steady-state concentration (PSSC) either trough and peak level in Type 2 diabetes mellitus patients with obesity and the impact of SLC22A1 gene organic cation transporter 1 (OCT) rs628031 A>G on PSSC of metformin.

Methods: Validated reversed-phase high-performance liquid chromatography method with ultraviolet detector was used to determine the metformin PSSC, as well as genotype variation was performed using the restriction fragment length polymorphisms-polymerase chain reaction method.

Results: A total of 13 patients were recruited from five Primary Health Centers in Yogyakarta Province of Indonesia. The results showed that the means of their trough and peak PSSC were $0.285 \pm 0.192$ and $1.175 \pm 0.814 \mu \mathrm{g} / \mathrm{ml}$, respectively. Only 10 patients $(77 \%)$ had peak PSSC within the plasma therapeutic level (PTL) of metformin, and 14-fold variability was observed for the peak PSSC. None of the patients achieved the PTL of metformin with regard to their trough PSSC. The PSSC of metformin was independent of the OCT1 genotype in rs628031 (A>G) 408M/V SLC22A1.

Conclusion: This study found a huge variability in the trough concentration of metformin ( $>100$-fold) and 14-fold for the peak PSSC, and no impact of a variant of rs628013 SLC22A1 OCT1 on metformin PSSC was revealed.

Keywords: Metformin, Steady-state pharmacokinetics, Obesity, Type 2 diabetes mellitus.

(C) 2017 The Authors. Published by Innovare Academic Sciences Pvt Ltd. This is an open access article under the CC BY license (http://creativecommons. org/licenses/by/4. 0/) DOI: http://dx.doi.org/10.22159/ajpcr.2017.v10i1.15252

\section{INTRODUCTION}

Metformin is the chosen oral antidiabetics to treat Type 2 diabetes mellitus (T2DM) patients with obesity [1]. In addition to its ability in reducing hemoglobin $\mathrm{A} 1 \mathrm{c}$ (HbA1c) level up to $1-2 \%$ and in improving insulin sensitivity, metformin has been known to be able to reduce body weight slightly. Those positive effects, along with lower incidence of hypoglycemia, have placed metformin in the first line of T2DM treatment, particularly, in patients with obesity [2,3]. However, the current dosing of metformin mostly refers to the drug-dosing data resulted from clinical studies on average-weight patients, and hence it is less clear to obtain metformin dosing-guidelines for the obese [4]. In addition, the results of Garber et al. demonstrated that the effectiveness of metformin as antidiabetic was correlated with its doses [5].

Metformin is available in the plasma largely as hydrophilic cation making it an unmetabolized drug with low plasma protein-binding so that its clearance is mostly affected only by cardiac output and renal function $[6,7]$. Therefore, the pharmacokinetic parameters of metformin could be changed in obese individuals, especially for the renal clearance because of increased glomerular filtration and tubular secretion $[7,8]$. Despite its wide therapeutic range, which is $0.75-5.00 \mu \mathrm{g} / \mathrm{ml}[9,10]$, it is suggested that the pharmacokinetic variability in an individualizing dosage of metformin should also be considered to gain optimal effectiveness without lactic acidosis [7]. In a clinical setting, the usual pharmacokinetic parameter to adjust dosage regimen in multiple drug administration is the drug steady-state level.

Several studies of plasma steady-state concentration (PSSC) of metformin have been conducted. A study of metformin trough PSSC in patients with unknown body mass index (BMI) was conducted on varying renal functions and doses [11]. Meanwhile, two studies investigated the low function of transporters, which is responsible for influx-efflux metformin in hepatocyte, and its influence on trough concentration of metformin $[12,13]$. However, studies on the peak steady-state level of metformin that could be used to estimate the maximum doses of metformin and its variability that could reflect the reduced-function of transporters mainly in the distribution process of metformin have never been conducted.

One of the transporters mediating the intestinal uptake of metformin [14] as well as the hepatic uptake of metformin across the sinusoidal membrane primarily is organic cation transporters 1 (OCT) [15]. Considerable works have been carried out with the OCT1 and its impact on both of pharmacokinetics as well as the response of metformin. Studies of genetic variants in specific population may illuminate important polymorphisms on metformin therapy [15]. Up to the present, investigation of OCT1 polymorphisms in Asian T2DM patients has been conducted in limited study. Results of Umamaheswaran et al. found a high frequency of the single nucleotide polymorphism (SNP) rs628031 OCT1 in Indian Population [16]. This variant could play an important role in pharmacokinetics and therapeutic response of metformin.

Alteration of pharmacokinetic parameters in obese patients, as well as reduced function of metformin transporters potentially, influence the obtained metformin PSSC, and further dose adjustment is required in case the intended PSCC and glycemic control are not reached. Considering the limited studies on PSSC of metformin in obese patients in Asian population as well as the existence of variant transporters of metformin that could affect both metformin pharmacokinetics and pharmacodynamics, this preliminary study aimed to determine the 
PSSC as well as the impact of rs628031 408M/V SLC22A1 OCT1 on metformin PSSC in Indonesian T2DM patients with obesity.

\section{METHODS}

T2DM patients with obesity, indicated by the BMI of $\geq 30 \mathrm{~kg} / \mathrm{m}^{2}$, were recruited from five Primary Health Centres during January-November 2015 in Yogyakarta province, Indonesia. Patients aged 35-60 years old, receiving a therapy of generic metformin at a dose of $500 \mathrm{mg}$ every $12 \mathrm{hrs}$ daily for at least 2 weeks as either a monotherapy or in combination with other antidiabetics, and willing to participate in the study were included. Patients with creatinine serum $>1.00 \mathrm{mg} / \mathrm{dL}$, history of chronic liver diseases, medication having potentially pharmacokinetic interaction with metformin such as cimetidine, furosemide, nifedipine [17], and systemic steroid treatment because of its glycemic uncontrolled effect [18] and patients not adhering to metformin therapy were excluded. Ethics approval for this research has been granted by the Faculty of Medicine - University of Gadjah Mada Ethics Committee. Written informed consent was obtained from all subjects.

\section{Steady state of metformin measurement}

To measure the trough PSSC of metformin, blood sample was taken immediately before administrating the next dose (pre-dose). Subsequently, the blood sample taken 3.5-4 hrs after metformin administration was used to measure the peak PSSC (post-dose). Determination of metformin plasma concentrations was carried out using validated reversed-phase high-performance liquid chromatography assay with a Sunfire ${ }^{\circledR} \mathrm{C}-18$ column, $4.6 \times 150 \mathrm{~mm} \times 5 \mu \mathrm{m}$ from waters, and SM7 injector with ultraviolet (UV) detector at $233 \mathrm{~nm}$ of wavelength. The mobile phase used a mixture of $45 \%$ acetonitrile and $55 \%$ buffer phosphate $\left(6 \mathrm{mM} \mathrm{KH}_{2} \mathrm{PO}_{4}, 6 \mathrm{mM}\right.$ sodium lauryl sulfate). All of the parameters for bioanalytical method have fulfilled the Guidance for Bioanalytical Method Validation from FDA. The linearity of the standard curve was (r) 0.9999 , and the accuracy value (\% difference) was $<15 \%$. Meanwhile, the value of precision (CV) was $<15 \%$. The obtained selectivity value (CV) was $<15 \%$, while the $\mathrm{CV}$ of recovery ranged $1.22-1.89 \%$ (unpublished observations). The obtained PSSCs were used to calculate metformin elimination rate by following one-compartment pharmacokinetic model [19]. The equation for elimination rate is [20]:

$\mathrm{K}_{\mathrm{el}}=\frac{\ln \mathrm{C}_{\text {max,ss }}-\ln \mathrm{C}_{\text {trough,ss }}}{\tau}$

\section{Genotyping}

Genomic DNA was isolated from whole blood, and genotyping for (rs rs628031 A $\rightarrow \mathrm{G}$ ) variations was performed using the restriction fragment length polymorphism-polymerase chain reaction (PCR) method. For PCR amplification, the following primers were used: Forward 5'-TTTCTTCAGTCTCTGACTCATGCC-3' and reverse 5'-AAAAAACTTTGTAGACAAAGGTAGCACC-3'. A thermo Scientific ${ }^{\circledR} 5020$ PCR was used for the amplification with PCR conditions as follows: Initial denaturation at $94^{\circ} \mathrm{C}$ for 5 minutes followed by 30 cycles of denaturation at $94^{\circ} \mathrm{C}$ for 30 seconds, annealing at $63^{\circ} \mathrm{C}$ for 45 seconds, primer extension for 1 minutes at $72^{\circ} \mathrm{C}$, and final extension at $72^{\circ} \mathrm{C}$ for 10 minutes. The PCR products were digested with MscI. The digestion of $397 \mathrm{bp}$ amplicon of rs628031 GG genotype resulted in $397 \mathrm{bp}$ fragments, and the AA genotype remained 210 and $187 \mathrm{bp}$, whereas the heterozygous genotype was 187, 210, $397 \mathrm{bp}$ fragments. The accuracy and reliability of all the genotyping methods have been confirmed by direct DNA sequencing in the previous study [16].

\section{Control glycemic assay}

For patients receiving metformin as a single therapy for 6 weeks, the parameters of glycemic responses were also assessed by measuring fasting blood glucose (FBG) and glycated albumin (GA) level. The GA was determined using ELISA reader ADVIA $^{\circledR}$, and UV/VIS spectrophotometry was utilized for the FBG. The intended FBG was defined as $<100 \mathrm{mg} / \mathrm{dl}$ while the GA was $<16 \%$ [21].

\section{Statistical analysis}

The results were expressed as mean \pm standard deviation. The peak PSSC of metformin was not normally distributed, thus the Mann-Whitney U-test was used to assess the difference mean among the groups, and the Spearman test was for the numerical correlation. As the nature of the study was slightly explorative, no sample size calculation was performed, but the size of the study population was estimated from a previous pharmacokinetic study of antibiotics in obese patients [22] as well as genetic variations in specific patients [13].

\section{RESULTS}

Thirteen T2DM patients were included in this study, in which all patients were female, and the patients' characteristics are presented in Table 1. Regarding the BMI, most patients were within the category of moderate obesity with age under 50-year-old. Most of the patients had estimated glomerular filtration rate (GFR) $\geq 100 \mathrm{ml} / \mathrm{min}$. The proportion of patients diagnosed as having T2DM for more or $<5$ years was quite similar. However, more patients used metformin twice daily as oral antidiabetics for more than 6 weeks before participating in this study, and so did patients with variant heterozygote Met/Val in SLC22A1.

In addition, metformin users had higher (1.98-fold) peak PSSC of metformin than patients with a combination therapy $(\mathrm{p}<0.05)$. It was not significantly different for the other characteristic groups in either trough or peak level of metformin.

Fig. 1 displays the achieved trough and peak PSSC of metformin for each patient participating in this study. The average sampling time for the determination of trough PSSC in this study was $12.18 \pm 0.24 \mathrm{hrs}$ after previous metformin administration, and the obtained PSSC ranged between 0.003 and $0.586 \mu \mathrm{g} / \mathrm{ml}$. Meanwhile, the average sampling time to determine the peak PSSC was $3.83 \pm 0.09 \mathrm{hrs}$ after the next administration of metformin, and the peak PSSC ranged $0.240-3.359 \mu \mathrm{g} / \mathrm{ml}$. From the obtained PSSC, an elimination rate of metformin was calculated accordingly, and it was found that the average elimination rate of metformin among the 13 patients was $0.15 \pm 0.11 / \mathrm{hr}$.

With regard to the trough PSSC, it was found that no patients reached the metformin plasma therapeutic level (PTL), and only 10 patients (77\%) whose peak PSSC reached metformin PTL were identified. However, the obtained PSSC in this study can be further used to estimate the adequate dose of metformin. By assuming that metformin followed the first order kinetics, the pharmacokinetic parameters of metformin such as half time, volume of distribution, and renal clearance were unchanged in patients. Hence, the recommended PTL of metformin with respect to the avoidance of lactic acidosis, which is PSSC $>5 \mu \mathrm{g} / \mathrm{mL}$, and the maximum

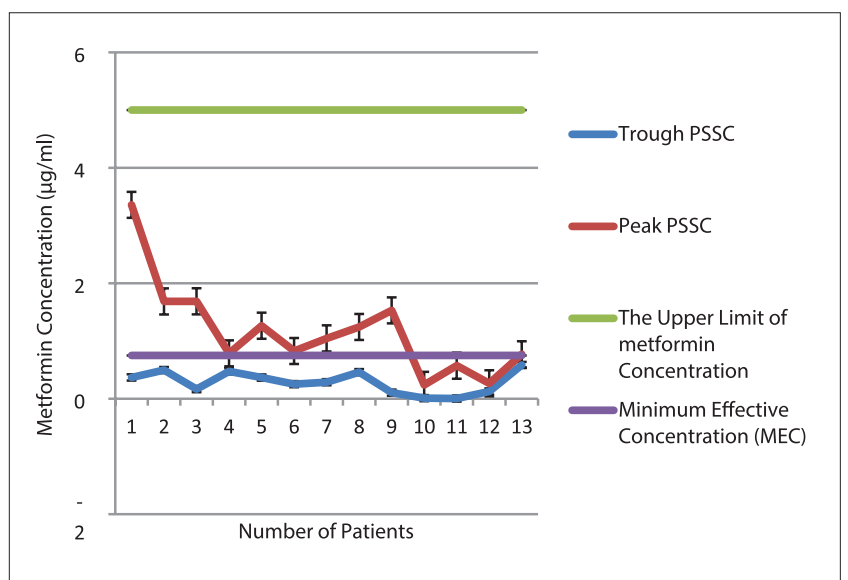

Fig. 1: Observed metformin plasma steady state concentration among 13 patients included in the study compared to the upper limit and minimum effective concentration of metformin 
dose of metformin for T2DM patients with obesity can be calculated accordingly. Given that the average peak PSSC obtained in this study was $1.175 \pm 0.814 \mu \mathrm{g} / \mathrm{mL}$, the estimated maximum dose of metformin for T2DM patients with obesity was $2513.83 \mathrm{mg} \infty 2500 \mathrm{mg}$ daily. Unfortunately, the minimum dose could not be calculated because of the large variability ( $>100$-fold) of the trough PSSC in this study [11]. As listed in Table 1, only 6 patients received metformin as a single therapy. For these 6 patients, an assessment of glycemic control was performed by using two parameters: The change of FBG and GA. It can be shown in Table 2 that no patient achieved the intended FBG, and only 2 patients had $\mathrm{GA}$ value within the defined range. In addition, due to the small number of cases in glycemic control assay, no formal analysis was conducted.

\section{DISCUSSION}

To conduct monitoring and evaluation of administered drug therapy is very important to ensure that the defined therapeutic responses have been achieved [23]. The most important aspect leading to the need of monitoring and evaluation for patients receiving metformin therapy is the variability of metformin pharmacokinetics and the risk of lactic acidosis in those patients with metformin plasma concentration $>5 \mu \mathrm{g} / \mathrm{mL}$ even in patients with normal renal function [24].

Other than the specific characteristics of obesity, the limited number of patients receiving metformin as a single therapy was the substantial reason for getting limited research subjects. Although several approaches have been conducted to obtain male patients for this study, no male patients were willing to participate; as a result, all recruited patients were female. Therefore, this study cannot rule out significant gender differences in the metformin PSSC of obese patients.
Based on the obtained PSSC in this study, the elimination rate was estimated at $0.15 \pm 0.11 / \mathrm{h}$, and it was higher than the elimination rate stated in literature, that is $0.112 / \mathrm{h}$ [9], probably indicating that metformin is excreted more effectively in obese individuals owing to increased GFR and tubular excretion than in patients with normal body weight.

One important finding of this study is that there were no patients reaching the PTL with regard to the trough PSSC. This means that the administered dose of metformin was inadequate to achieve the intended therapeutic level, which potentially leads to the failure of obtaining defined therapeutic responses [23]. Considering that the half-life of metformin is approximately 5 hrs in T2DM patients with normal renal function [25], administering conventional dosage form of metformin every $12 \mathrm{hrs}$ (2.4fold) resulted in trough PSSC [20] that is under the minimum effective concentration of metformin. Researches on pharmacokinetics and pharmacodynamics modeling exhibit a correlation between metformin plasma levels and glycemic responses [26]. In addition, doses were a dependent of the glycemic response of metformin [5].

Furthermore, this research found that only 10 patients (77\%) had peak PSSC within the PTL while none of the patients had metformin levels $>5 \mathrm{mg} / \mathrm{mL}$ indicating low risk of lactic acidosis. Although the plasma level of metformin can be used to estimate the occurrence of lactic acidosis, other parameters such as symptoms of nausea and fatigue as well as abnormalities in lactate levels (plasma lactate levels $>4 \mathrm{mmol} / \mathrm{L}$ and/or $\mathrm{pH}<7.37$ ) should also be confirmed [27]. PSCC $<5 \mathrm{mg} / \mathrm{mL}$ was observed by Duong et al. in dosing sumulations for patients with $\mathrm{ClCr}$ $30 \mathrm{ml} / \mathrm{min}$ receiving metformin $1000 \mathrm{mg}$ daily [10]. Commonly, the risk of lactic acidosis due to metformin administration increases when the

Table 1: Characteristics of patients

\begin{tabular}{|c|c|c|c|c|c|}
\hline Characteristics & Number (\%) & Trough PSSC \pm SD $(\mu \mathrm{g} / \mathrm{ml})$ & $\mathbf{p}$ & Peak PSSC \pm SD $(\mu \mathrm{g} / \mathrm{ml})$ & $\mathbf{p}$ \\
\hline \multicolumn{6}{|l|}{ Age (years) } \\
\hline$<50$ & $8(61.5)$ & $0.2935 \pm 0.1611$ & \multirow[t]{2}{*}{0.850} & $1.1291 \pm 0.9822$ & \multirow[t]{2}{*}{0.464} \\
\hline$\geq 50$ & $5(38.5)$ & $0.2714 \pm 0.2553$ & & $1.2496 \pm 0.5361$ & \\
\hline \multicolumn{6}{|l|}{ BMI $\left(\mathrm{kg} / \mathrm{m}^{2}\right)$} \\
\hline $30-35$ & $10(76.9)$ & $0.2739 \pm 0.1732$ & \multirow[t]{2}{*}{0.721} & $1.0912 \pm 0.4795$ & \multirow{2}{*}{0.612} \\
\hline$\geq 35$ & $3(23.1)$ & $0.3220 \pm 0.2904$ & & $1.4563 \pm 1.6689$ & \\
\hline \multicolumn{6}{|l|}{$\mathrm{eGFR}\left(\mathrm{ml} / \mathrm{min} / 1.73 \mathrm{~m}^{2}\right)$} \\
\hline $70-100$ & $4(30.8)$ & $0.2235 \pm 0.1841$ & \multirow[t]{2}{*}{0.466} & $1.2930 \pm 0.6879$ & \multirow[t]{2}{*}{0.280} \\
\hline$\geq 100$ & $9(69.2)$ & $0.3123 \pm 0.2001$ & & $1.1232 \pm 0.8978$ & \\
\hline \multicolumn{6}{|l|}{ Duration of T2DM (years) } \\
\hline$<5$ & $7(53.8)$ & $0.2520 \pm 0.1816$ & \multirow[t]{2}{*}{0.528} & $1.1566 \pm 1.0248$ & \multirow[t]{2}{*}{0.475} \\
\hline$\geq 5$ & $6(46.2)$ & $0.3235 \pm 0.2142$ & & $1.1975 \pm 0.5729$ & \\
\hline \multicolumn{6}{|l|}{ Duration of metformin (weeks) ${ }^{a}$} \\
\hline $2-<6$ & $6(46.2)$ & $0.2498 \pm 0.2035$ & \multirow[t]{2}{*}{0.565} & $1.3250 \pm 1.1191$ & \multirow[t]{2}{*}{0.886} \\
\hline$\geq 6$ & $7(53.8)$ & $0.3151 \pm 0.1929$ & & $1.0473 \pm 0.4893$ & \\
\hline \multicolumn{6}{|l|}{ Metformin regimen } \\
\hline Single & $6(46.2)$ & $0.3543 \pm 0.1275$ & \multirow[t]{2}{*}{0.245} & $1.6020 \pm 0.9466$ & \multirow[t]{2}{*}{$0.046^{*}$} \\
\hline In combination with other antidiabetics & 7 (53.8) & $0.2256 \pm 0.2270$ & & $0.8099 \pm 0.4899$ & \\
\hline \multicolumn{6}{|l|}{ Genotype $^{b}$} \\
\hline GG & $3(25.0)$ & $0.2433 \pm 0.2361$ & \multirow[t]{2}{*}{0.862} & $1.3693 \pm 0.9226$ & \multirow[t]{2}{*}{0.166} \\
\hline $\mathrm{AG}$ & $9(75.0)$ & $0.2654 \pm 0.1707$ & & $0.7290 \pm 0.1366$ & \\
\hline
\end{tabular}

${ }^{*} \mathrm{p}<0.05,{ }^{\mathrm{a}}$ At dose of $500 \mathrm{mg}$ every $12 \mathrm{hrs},{ }^{\mathrm{b}} 1$ patient with wild-type AA. PSSC: Plasma steady-state concentration, T2DM: Type 2 diabetes mellitus, eGFR: Estimated glomerular filtration rate, BMI: Body mass index

Table 2: Glycemic control responses among 6 patients after receiving a single therapy of metformin 500 mg every 12 hrs

\begin{tabular}{llllll}
\hline S.No. & Genotype & Baseline of FBG $\mathbf{( m g / d L )}$ & FBG after $\mathbf{6}$ weeks $\mathbf{( m g / d L )}$ & Baseline of GA (\%) & GA after 6 weeks (\%) \\
\hline 1 & AG & 108 & 105 & 15.96 & 15.45 \\
2 & AG & 135 & 127 & 17.89 & 18.39 \\
3 & GG & 152 & 131 & 16.59 & 15.53 \\
4 & AG & 119 & 268 & 24.37 & 19.92 \\
5 & GG & 175 & 189 & 22.27 & 22.1 \\
6 & AG & 199 & 173 & 23.21 \\
\hline
\end{tabular}

Spearman test: Between baseline FBG and GA ( $p=0.072, r=0.771$ ); between FBG and GA after metformin administered for 6 weeks ( $<<0.05$, $r=0.886$ ). FBG: Fasting blood glucose, GA: Glycated albumin 
tissue suffers from hypoxia as in the case of myocardial infarction, acute left heart failure or septicemia as well as renal or liver failure $[27,28]$.

Although this research was unable to estimate the metformin dose based on a pharmacokinetics-pharmacodynamics profile due to the limited number of patients, the findings can be considered for a maximum dose recommendation of metformin in T2DM patients with obesity. The maximum twice daily dosing of metformin in T2DM patients with obesity based on the observed peak PSSC in this research was $2500 \mathrm{mg}$ daily, and this is almost similar to the recommended maximum dose of metformin for adult patients with average body weight stated in the literature $(2550 \mathrm{mg} /$ day) [18].

In addition, the unachieved PTL in regard to the trough PSSC of metformin in this research indicated that the administered daily dose of $1000 \mathrm{mg}$ metformin was inadequate, and this was further proved by a decrease of FBG only in 4 patients and 3 patients for the GA after metformin administration for 6 weeks. In a study involving 45 obese patients with T2DM, a significant reduction of FBG was observed after treated with metformin $500 \mathrm{mg}$ twice daily for 1 year [29]. Yasser et al. found $18.3 \%$ reduction of $\mathrm{HbA} 1 \mathrm{c}$ in 10 patients with newly diagnosed of T2DM after receiving $1000 \mathrm{mg}$ metformin [30]. The difference on the duration of metformin administration, the baseline of glycemic index, as well as involved patients with normal renal function could be the reasons of this present results. In addition, since GA reflects a shorter glycemic control resulted from shorter life span of albumin (17 days) compared to erythrocyte [31], make GA is an intermediate-term glycation index. A study including 18 patients with T2DM showed a GA reduction up to 8.1\% after being administered with metformin and monitored every 4 weeks for 24 weeks [32]. Because of limited sample size, no statistical analysis for the glycemic control was performed in this study.

A higher peak PSSC of metformin instead of trough level in variant homozygote GG group was found in this study ( $\mathrm{p}=0.166$ ). OCT1 (gene SLC22A1) is expressed on the basolateral membrane and cytoplasm of the enterocytes and predominantly found in hepatocytes [14]. Since the variant homozygote have greater impact to protein function, thereby OCT1 reduced-function may contribute to a decrease in influx of metformin to hepatocytes and result in higher peak PSSC of metformin than in heterozygote variant group in this study.

Meanwhile, OCT1 is also expressed on the apical and subapical domain side of both the proximal and the distal tubules in the kidney [33], so it plays an important role in the renal clearance of metformin. In the present study, there was no impact on metformin PSSC of the frequent OCT1 SNP rs628031 (408M/V), which was contrary to the result of Shikata et al. that demonstrated lower expression of mRNA in OCT1 in the hepatocyte of human liver with 408M/V variant in SLC22A1 [34]. Since OCT1 is the main transporter responsible in the influx process of metformin into hepatocyte, the existence of variance leads to a reduced function of the transporter. Consequently, metformin in a level higher than that in wildtype patients was observed. The smaller size of sample and number of observation could explain the insignificant difference in these findings.

This present study also revealed a quite large number of frequency of SNP rs628031 OCT1 in Indonesian T2DM patients, so further studies about the impact of this variant on gastrointestinal side effect and risk of hypoglycemia events as already reported in several studies $[35,36]$ should be conducted for Indonesian population. As this research also found a variability of metformin peak PSSC, as well as a significantly higher level in metformin users, a pharmacogenetic and detailed pharmacokinetic study on monotherapy compared to combination therapy with sulfonylurea, are required to improve the therapeutic management of T2DM patients receiving metformin.

\section{CONCLUSION}

This study found a huge variability in the trough concentration of metformin $(>100$-fold) and 14-fold for the peak PSSC. The PSSC of metformin was independent of the OCT1 genotype in rs628031408M/V SLC22A1. This study indicated that the maximum doses of metformin were relatively similar to what is listed in the literature; also, the prescription of metformin with twice daily dose of $500 \mathrm{mg}$ was inadequate for T2DM patients with obesity. However, due to the small size of samples, further studies are needed to draw firm conclusions.

\section{ACKNOWLEDGEMENT}

The research was funded by the Directorate of Research and Community Service and PSPA Universitas Islam, Indonesia. The authors have declared no conflict of interest.

\section{REFERENCES}

1. Hollander P. Anti-diabetes and anti-obesity medications: Effects on weight in people with diabetes. Diabetes Spectr 2007;20(3):159-65.

2. Golay A. Metformin and body weight. Int J Obes (Lond) 2008(1);32:61-72.

3. Malin SK, Kashyap SR. Effects of metformin on weight loss: Potential mechanisms. Curr Opin Endocrinol Diabetes Obes 2014;21(5):323-9.

4. Green B, Duffull SB. What is the best size descriptor to use for pharmacokinetic studies in the obese? $\mathrm{Br} \mathrm{J}$ Clin Pharmacol 2004;58(2):119-33.

5. Garber AJ, Duncan TG, Goodman AM, Mills DJ, Rohlf JL. Efficacy of metformin in type II diabetes: Results of a double-blind, placebocontrolled, dose-response trial. Am J Med 1997;103(6):491-7.

6. Ghobadi C, Johnson TN, Aarabi M, Almond LM, Allabi AC, Rowland-Yeo $\mathrm{K}$, et al. Application of a systems approach to the bottom-up assessment of pharmacokinetics in obese patients: Expected variations in clearance. Clin Pharmacokinet 2011;50(12):809-22.

7. Graham GG, Punt J, Arora M, Day RO, Doogue MP, Duong JK, et al. Clinical pharmacokinetics of metformin. Clin Pharmacokinet 2011:50(2):81-98

8. Ghose R. Clinical consequences of altered drug disposition in obesity. J Clin Trials 2012;2:e107.

9. Ritscel W, Kearns G. Handbook of Basic Pharmacokinetics including Clinical Applications. $6^{\text {th }}$ ed. Washington DC: American Pharmacist Association; 2004

10. Duong JK, Kumar SS, Kirkpatrick CM, Greenup LC, Arora M, Lee TC, et al. Population pharmacokinetics of metformin in healthy subjects and patients with Type 2 diabetes mellitus: Simulation of doses according to renal function. Clin Pharmacokinet 2013;52(5):373-84

11. Frid A, Sterner GN, Löndahl M, Wiklander C, Cato A, Vinge E, et al. Novel assay of metformin levels in patients with type 2 diabetes and varying levels of renal function: Clinical recommendations. Diabetes Care 2010;33(6):1291-3

12. Christensen MM, Brasch-Andersen C, Green H, Nielsen F, Damkier P, Beck-Nielsen $\mathrm{H}$, et al. The pharmacogenetics of metformin and its impact on plasma metformin steady-state levels and glycosylated hemoglobin Alc. Pharmacogenet Genomics 2011;21(12):837-50.

13. Stage TB, Damkier P, Pedersen RS, Christensen MM, Christiansen L, Christensen $\mathrm{K}$, et al. A twin study of the trough plasma steadystate concentration of metformin. Pharmacogenet Genomics 2015;25(5):259-62.

14. Müller J, Lips KS, Metzner L, Neubert RH, Koepsell H, Brandsch M. Drug specificity and intestinal membrane localization of human organic cation transporters (OCT). Biochem Pharmacol 2005;70(12):1851-60.

15. Gong L, Goswami S, Giacomini KM, Altman RB, Klein TE. Metformin pathways: Pharmacokinetics and pharmacodynamics. Pharmacogenet Genomics 2012;22(11):820-7.

16. Umamaheswaran G, Praveen RG, Arunkumar AS, Das AK, Shewade DG, Adithan C. Genetic analysis of OCT1 gene polymorphisms in an Indian population. Indian J Hum Genet 2011:17(3):164-8.

17. Rojas LB, Gomes MB. Metformin: An old but still the best treatment for Type 2 diabetes. Diabetol Metab Syndr 2013;5(1):6.

18. American Pharmacist Association. Drug Information Handbook. $20^{\text {th }}$ ed. Ohio, USA: Lexi-Comp; 2012.

19. Bardin C, Nobecourt E, Larger E, Chast F, Treluyer JM, Urien S. Population pharmacokinetics of metformin in obese and nonobese patients with Type 2 diabetes mellitus. Eur J Clin Pharmacol 2012;68(6):961-8

20. Shargel L, Wu-Pong S, Yu AB. Applied Biopharmaceutics and Pharmacokinetics. New York: Appleton \& Lange Reviews, McGraw Hill; 2005

21. Roohk HV, Zaidi AR. A review of glycated albumin as an intermediate 
glycation index for controlling diabetes. J Diabetes Sci Technol 2008;2(6):1114-21

22. Chen M, Nafziger AN, Drusano GL, Ma L, Bertino JS Jr. Comparative pharmacokinetics and pharmacodynamic target attainment of ertapenem in normal-weight, obese, and extremely obese adults. Antimicrob Agents Chemother 2006;50(4):1222-7.

23. Gross AS. Best practice in therapeutic drug monitoring. Br $\mathrm{J}$ Clin Pharmacol 2001;52 Suppl 1:5S-10S.

24. Bruijstens LA, van Luin M, Buscher-Jungerhans PM, Bosch FH. Reality of severe metformin-induced lactic acidosis in the absence of chronic renal impairment. Neth J Med 2008;66(5):185-90.

25. Graham GG, Punt J, Arora M, Day RO, Doogue MP, Duong JK, et al. Clinical pharmacokinetics of metformin. Clin Pharmacokinet 2011;50(2):81-98.

26. Hong Y, Rohatagi S, Habtemariam B, Walker JR, Schwartz SL, Mager DE. Population exposure-response modeling of metformin in patients with Type 2 diabetes mellitus. J Clin Pharmacol 2008;48(6):696707.

27. Tahrani AA, Varughese GI, Scarpello JH, Hanna FW. Metformin, heart failure, and lactic acidosis: Is metformin absolutely contraindicated? BMJ 2007;335(7618):508-12.

28. Jones GC, Macklin JP, Alexander WD. Contraindications to the use of metformin. BMJ 2003;326(7379):4-5.

29. Saveetha V, Sundaram IS, Shanmugasundaram P. A prospective study on the efficacy of metformin in obese and non-obese patients with polycyctic ovary syndrome. Asian J Pharm Clin Res 2016;9 Suppl 1:145-7.
30. Yaseer MK, Abbas MR, Saba H. The effect of metformin on cytokines in Iraqi patients with type 2 diabetes. Innovare J Med Sci 2013;1(3):4-10.

31. Yang C, Li H, Wang Z, Zhang W, Zhou K, Meng J, et al. Glycated albumin is a potential diagnostic tool for diabetes mellitus. Clin Med (Lond) 2012;12(6):568-71.

32. Sumitani S, Morita S, Deguchi R, Hirai K, Mukai K, Utsu Y, et al. Metformin decreases glycated albumin to glycated haemoglobin ratio in patients with newly diagnosed Type 2 diabetes. Ann Clin Biochem 2015;52(1):76-81.

33. Tzvetkov MV, Vormfelde SV, Balen D, Meineke I, Schmidt T, Sehrt D, et al. The effects of genetic polymorphisms in the organic cation transporters OCT1, OCT2, and OCT3 on the renal clearance of metformin. Clin Pharmacol Ther 2009;86(3):299-306.

34. Shikata E, Yamamoto R, Takane H, Shigemasa C, Ikeda T, Otsubo K, et al. Human organic cation transporter (OCT1 and OCT2) gene polymorphisms and therapeutic effects of metformin. J Hum Genet 2007;52(2):117-22.

35. Tarasova L, Kalnina I, Geldnere K, Bumbure A, Ritenberga R, Nikitina-Zake L, et al. Association of genetic variation in the organic cation transporters OCT1, OCT2 and multidrug and toxin Extrusion 1 transporter protein genes with the gastrointestinal side effects and lower BMI in metformin-treated Type 2 diabetes patients. Pharmacogenet Genomics 2012;22(9):659-66.

36. Klen J, Goricar K, Janež A, Dolžan V. The role of genetic factors and kidney and liver function in glycemic control in Type 2 diabetes patients on long-term metformin and sulphonylurea cotreatment. Biomed Res Int 2014;2014:934729. 